\title{
Die Objektivierung der Dinge. Wissenspraktiken im mathematisch-naturwissenschaftlichen Schulunterricht Objectifying Things. Epistemic Practices in Science Education
} Tobias Röhl*

Institut für Soziologie, Johannes Gutenberg-Universität Mainz, Jakob-Welder-Weg 12, 55128 Mainz, Germany. tobias.roehl@uni-mainz.de

\begin{abstract}
Zusammenfassung: Auf Grundlage ethnografischer Daten beschäftigt sich der Aufsatz mit Experimenten und Anschauungsobjekten im mathematisch-naturwissenschaftlichen Schulunterricht der Sekundarstufe. Die Unterrichtsteilnehmer transformieren dabei vieldeutige Dinge in eindeutige und von ihnen als unabhängig gefasste Objekte. Diese Objektivierung der Dinge erfolgt im Schulunterricht in drei Phasen: Nach der Vorbereitung im schulischen Archiv werden die Dinge bei der Durchführung von Experimenten im Unterrichtsraum in Aktion versetzt und schließlich in die sprachliche bzw. schriftliche Form an der Tafel und in den Heften überführt. Diese praktische Transformationsleistung ist durch eine asymmetrische Verteilung von Rede- und Manipulationsrechten sowie die Gestaltung der Dinge abgesichert und baut auf ein distanziertes (meist visuelles) Erkenntnismodell. Damit üben die Unterrichtsteilnehmer auch eine zwischen kulturellen Subjekten und materiellen Objekten unterscheidende gesellschaftliche Haltung ein.
\end{abstract}

Schlagworte: Wissenssoziologie; Materialität; Soziologie der Dinge; Ethnografische Unterrichtsforschung; Physikunterricht.

Summary: This paper presents ethnographic data on the use of learning materials in science education at the secondary school level. In science classes teachers and students transform ambiguous things into clearly defined and independent objects. This objectification of things is achieved in the course of three distinct phases: first, learning materials are prepared in an experimental set-up in designated rooms; second, they are put into practice when experiments are conducted; and, third, they are transferred to language and signs on the blackboard and in notebooks. This practical achievement is stabilized via an asymmetric distribution of activities and the design of learning materials. In relying on an epistemological paradigm that emphasizes visual appraisal at a distance science classes perpetuate a perspective in which cultural subjects and material objects are clearly distinguished.

Keywords: Sociology of Knowledge; Materiality; Sociology of Things; Educational Ethnography; Science Education.

\section{Einleitung}

Naturwissenschaftliches Wissen tritt umso gesicherter auf, je mehr die mundanen Praktiken seiner Herstellung in den schriftlichen Berichten und Veröffentlichungen ausgeblendet bleiben (Knorr-Cetina 1984; Latour \& Woolgar 1979). Damit einher geht eine ,Härtung' der Forschungsobjekte: Aus zunächst undefiniertem Rauschen wird z. B. ein Pulsar, der im wissenschaftlichen Bericht als immer schon in dieser Weise vorhandenes Objekt erscheint (Garfinkel et al. 1981). Wie dieses konkret und praktisch geschaffen wurde, gilt als unerheblich es erscheint als von den epistemischen Praktiken seiner Herstellung unabhängiges Objekt. Diese ob-

\footnotetext{
* Für ihre hilfreichen Anmerkungen und Kommentare danke ich den GutachterInnen und HerausgeberInnen sowie Herbert Kalthoff, Bettina Grimmer und Björn Krey.
}

jektivierende Sicht ist nicht auf das naturwissenschaftliche Labor beschränkt; Latour (2008) hat sie als ein Charakteristikum modernen Denkens herausgearbeitet. Als Mitglieder westlicher Gesellschaften unterscheiden wir klar zwischen Menschen und deren Sozialität auf der einen und Technik und Materie auf der anderen Seite. Ein zentraler Ort, an dem wir einer solchen objektivierenden Haltung in verdichteter Form begegnen, ist die Schule und dort insbesondere der mathematischnaturwissenschaftliche Unterricht. Gerade in diesen Schulfächern setzt man auf eine Reihe von Dingen, die Sachverhalte und Phänomene veranschaulichen sollen; geometrische Modelle im Mathematikunterricht und die zahlreichen Versuchsaufbauten des Physikunterrichts sind Beispiele hierfür. Als schulische Vertreter der hard sciences zielen diese Schulfächer in besonderer Weise auf die Objektivierung der Dinge. In den mathematisch-naturwissenschaft- 
lichen Fächern werden aus vieldeutigen Dingen klar umrissene Objekte gemacht, die uns scheinbar unabhängig gegenüberstehen.

Ziel des Aufsatzes ist es, diese schulische Objektivierung der Dinge empirisch als das Ergebnis einer praktischen Transformationsleistung nachzuzeichnen. Mit Dingen sind relativ klar umrissene materielle Entitäten gefasst, die in praktischen „Verweisungszusammenhängen “ (Heidegger 2006: 63-88) stehen. Objekte hingegen sind spezifische Dinge, die von den Akteuren als von ihren Praktiken unabhängig und ihnen klar gegenübergestellt behandelt werden. Letzteres ist als Ergebnis einer spezifischen objektivierenden Umgangsweise mit Dingen zu verstehen. Die Objektivierung ist dabei ein gradueller Prozess, der sich auch jenseits des Schulunterrichts oder des Labors finden lässt: Jedes Ding ist bereits durch den jeweiligen praktischen Kontext sowie durch seine Gestalt in der Vieldeutigkeit seiner stofflichen Materialien eingeschränkt (vgl. Ingold 2007). Gleichwohl findet sich beim Ding eine praktische Verschränkung von Sozialität und Materialität. Beim Objekt wird diese Verschränkung hingegen geleugnet und die Objektivierung findet ihren Höhepunkt. Auch die hier vorgestellte Forschung muss eine objektivierende Haltung gegenüber der stofflichen Materialität einnehmen, um Dinge überhaupt beobachten zu können (vgl. Neumann 2012: 172-174). Allerdings gewinnt sie mit der Idee der Transformation ein für die Vieldeutigkeit und Wandelbarkeit der Dinge sensitives Vokabular.

Das Interesse an der praktischen Transformationsleistung reagiert auf zwei Desiderate: Erstens weiß die Wissenschaftsforschung nur wenig über die Tragweite wissenschaftlichen Wissens jenseits des Labors bzw. der scientific community. Dies ist insbesondere angesichts eines vermehrt geäußerten allgemeinsoziologischen Anspruchs bedauerlich (etwa bei Latour 2007; Knorr-Cetina 2007). Ein Blick in alltäglichere Bereiche kann deshalb zeigen, was mit kanonisiertem wissenschaftlichen Wissen und der dazugehörigen Weltsicht außerhalb der Wissenschaft passiert. Ein vielversprechendes Forschungsfeld ist hier die qualitative Unterrichtsforschung, die unter anderem den unterschiedlichen interaktionalen Formaten der Wissensvermittlung nachgeht (etwa Breidenstein 2006; Kalthoff 1997; Mehan 1979). Die Frage ist dann, welche „Modulationen“ (Goffman 1980: 52ff.) vorgenommen werden müssen, damit die objektivierende Transformation vor einem nur bedingt daran beteiligten Publikum (der Klasse) gelingen kann. So kann im Physikunterricht beispielsweise kaum von Experimenten im engeren
Sinne gesprochen werden. Stattdessen finden wir einen „inszenierte[n] Erkenntnisprozess“ (Erlemann 2004: 66) vor, bei dem Novizen unter Anleitung einer initiierten Lehrperson kanonisiertes Wissen kennenlernen. Dies widerspricht der prinzipiellen Ergebnisoffenheit des wissenschaftlichen Experiments. Zweitens hat die qualitative Unterrichtsforschung mit ihrem Fokus auf Interaktion und Kommunikation die materielle Dimension der Bildung lange vernachlässigt. Von der Wissenschaftsforschung kann die qualitative Unterrichtsforschung deshalb lernen, Fragen nach dem praktischen Status von Dingen zu stellen. Damit schließt die hier vorgestellte Forschung an eine Reihe neuerer Studien an, die sich der Materialität von Bildung und Erziehung zuwenden (z. B. Priem et al. 2012; Nohl \& Wulf 2013).

Mit der analytischen Re-Deskription eines (schulisch) alltäglichen Phänomens leistet der Aufsatz also dreierlei: (1) Der qualitativen Unterrichtsforschung ermöglicht ein solches Vorgehen eine Erweiterung des Fokus um die epistemisch-materielle Dimension; die jeweilige „Fachkultur“ (Lüders 2007) beruht dann nicht nur auf faktischem Wissen und „interactional skills“ (Mehan 1979: 139), sondern auch auf einem je spezifischen praktischen (vorsprachlichen) Umgang mit Dingen, der im Fall des mathematisch-naturwissenschaftlichen Schulunterrichts in einer objektivierenden Transformation besteht; (2) über den Schulunterricht hinaus zeigt der Aufsatz, wie eine bestimmte Haltung gegenüber den Dingen gesellschaftlich umgesetzt wird; (3) schließlich liefert er aber auch einen Beitrag zur Diskussion um die angemessene Berücksichtigung der Dinge in der Sozialtheorie.

Die hier eingenommene Haltung entspricht insofern einem „empirischen Konstruktivismus“ (KnorrCetina 1989: 91-95), als ontologische (etwa Heidegger 1959) oder psychologische Fragen (etwa Piaget 1992) nach der Objektkonstitution als praktische Probleme der Teilnehmer reformuliert werden. Dementsprechend werden Wahrheits- und Wirklichkeitsansprüche bezüglich dieser Objekte als Teilnehmerkategorie verstanden und im beobachtbaren praktischen Umgang mit den Dingen aufgesucht. Dinge werden aus der Teilnehmersicht also dann als unabhängige, immer schon so existierende Objekte begriffen, wenn sie in entsprechender Weise gehandhabt werden. Anders als die klassische phänomenologische Wissenschaftskritik (Gurwitsch 1974) verstehe ich die Objektivierung lebensweltlicher Dinge deshalb nicht als beklagenswerten Verlust, sondern als praktische kulturelle Leistung der Unterrichtsteilnehmer. 
Methodisch wird hier auf die Idee einer „prozeduralen Ethnografie“ (Kalthoff 2003) zurückgegriffen, die sich in situ, also zu den Teilnehmern in ihren Lokalitäten begibt. Anders als eine Ethnografie, die vor allem auf das Gespräch mit Informanten setzt, ermöglicht die so vollzogene teilnehmende Beobachtung einen Zugang zu den Gebrauchspraktiken in ihrem tatsächlichen Ablauf. Damit öffnet sich der ethnografischen Forschung auch die oft stillschweigend vorgenommene Transformation der Dinge, die sich gerade auch in nicht sprachlichen Gebrauchsweisen zeigt. Der Schulunterricht erweist sich dabei als besonders dankbares Feld, da die Teilnehmer didaktischer Praktiken in besonderem Maße explizit machen müssen, was andernorts implizit bleiben kann (vgl. ten Have 2004: 154). Die hier diskutierten empirischen Daten sind auf der Grundlage von mehrmonatigen ethnografischen Feldaufenthalten an drei Gymnasien und einer Gesamtschule in Baden-Württemberg und RheinlandPfalz in den Jahren 2009-2011 entstanden. Die untersuchten Schulstunden folgten einem ,klassisch lehrerzentrierten Format. Insgesamt habe ich 72 Unterrichtsstunden in den Klassenstufen 5-12 in den Fächern Mathematik und Physik beobachtet. Neben Beobachtungsprotokollen bilden Fotografien, Audioaufzeichnungen und Interviews mit Lehrkräften und Schülern den Datenkorpus.

Zunächst werden verschiedene theoretische $\mathrm{Zu}$ gänge vorgestellt, mit denen sich Dinge als wandelbare Entitäten fassen lassen (2.). Danach zeige ich die verschiedenen Schritte und praktischen Verfahren auf, mit denen die Unterrichtsteilnehmer schulische Dinge handhaben und dadurch in Objekte transformieren (3.). Daran anschließend wird diese Transformationsleistung vor dem Hintergrund allgemeinerer Überlegungen zur Bedeutung materieller Dinge und ihrer Transformation in der (Unterrichts-)Praxis diskutiert (4.).

\section{Theoretische Vorüberlegungen}

Konzeptionelle Anschlussmöglichkeiten für die hier vorgestellte Forschung finden sich in der Debatte um die „New Materialisms“ (Coole \& Frost 2010) in den Kultur- und Sozialwissenschaften (Balke et al. 2012; Kalthoff et al. 2015). Kennzeichnend für diese neuen Ausprägungen einer materialistischen Haltung ist die Ablehnung eines essentialistischen Konzepts von Materie im Allgemeinen und Dingen im Besonderen. Dinge sind hier nicht qua ihrer Natur mit unverrückbaren Eigenschaften ausgestattet und als jedem Kontext enthobene Entitäten ge- dacht, sondern stets in Relation zu sehen. Erst in spezifischen Kontexten erhalten Dinge ihre je wirksamen Eigenschaften. Gegen eine dekontextualisierende Haltung kann man in der Literatur drei Rekontextualisierungen bzw. Relationierungen ausmachen.

(1) Arrangements: Eine Möglichkeit, die Wirkmacht der Dinge zu relationieren, besteht darin, sie als Teil eines Arrangements aus Dingen und Menschen zu begreifen. Prominent ist hier die AkteurNetzwerk-Theorie (ANT; Callon 1986; Latour 2001), die materielle Artefakte als Bestandteile heterogener Netzwerke fasst. Annahmen über den ontologischen Status der Bestandteile solcher Netzwerke sind bewusst zurückgestellt. Entsprechend minimal ist deshalb der Akteursbegriff gefasst: „jedes Ding, das eine gegebene Situation verändert, indem es einen Unterschied macht, [ist] ein Akteur" (Latour 2007: 123; H.i.O.). Als Akteure zählen neben Dingen dementsprechend auch Engel, Viren oder Naturphänomene. Handlungsträgerschaft wird hier nicht einzelnen Entitäten zugeschrieben, sondern im Verbund unterschiedlicher menschlicher und nicht-menschlicher Akteure gesucht. Dabei sind auch räumlich und zeitlich weit entfernte Orte und Institutionen mittelbar eingebunden. Über die architektonische Gestaltung eines Postschalters sind beispielsweise die Verwaltungsebene der Post sowie das Architekturbüro in der Interaktion zwischen Kunde und Postangestelltem anwesend und bestimmen diese mit (Latour 2001: 247f.). Mit der ANT werden das Zustandekommen und die Wirkmacht weitreichender Ketten von miteinander verbundenen Akteuren für die Forschung fassbar. Aus Sicht der technografischen Techniksoziologie wird damit jedoch der konkrete praktische Beitrag einzelner Dinge diffus und es fehlt an einem differenzierten Vokabular zur Bestimmung der dinglichen Wirkmacht (Rammert 2008; Rammert \& Schulz-Schaeffer 2002; Schubert 2011: 179). Dort setzt man deshalb darauf, die tatsächliche Verteilung der Handlungsträgerschaft in sozio-technischen Konstellationen nachzuzeichnen. Möglich macht dies ein ,gradualisierter Handlungsbegriff“, der zwischen verschiedenen Graden unterscheidet: von der einfachen „verändernden Wirksamkeit" (Kausalität) über die Fähigkeit des „auch-anders-handeln-Könnens“ (Kontingenz) bis hin zur „intentionalen Erklärung“ (Intentionalität; Rammert 2008: 356). In der qualitativen Unterrichtsforschung findet sich die Idee des Arrangements in Forschungen, die sich unter Rückgriff auf die ANT für die komplexen und weitreichenden Netzwerke aus Menschen und Technik interessieren 
(Fenwick \& Edwards 2010). So können beispielsweise die wechselseitigen Anpassungsprozesse bei der Einführung neuer Technologien nachgezeichnet (Sørensen 2009) oder die räumliche Verteilung von Bildungspraktiken beschrieben werden (Nespor 1994).

(2) Körper: Eine weitere Möglichkeit, Dinge zu relationieren, ist, nach ihrem Bezug zum menschlichen Körper zu fragen. Soziologische und technikphilosophische Studien greifen auf phänomenologische Überlegungen zurück, um die leiblich-sinnliche Verwicklung von Menschen, Dingen und Welt nachzuzeichnen (etwa Frers 2007; Gugutzer 2012; Introna et al. 2008; Lindemann 2009). Ihde (1990) weist beispielsweise darauf hin, dass technische Artefakte unterschiedlich für menschliche Körper präsent sind und zwischen Welt und Menschen vermitteln: als transparente leibliche Prothese (Brille), als opakes Gegenüber (Roboter, Spielzeug), als Träger $\mathrm{zu}$ interpretierender Zeichen (Thermometer) oder als unauffällige Infrastruktur (Klimaanlage). Ihre Wirkmacht besteht hier weniger in einem Zwang, als vielmehr darin, in ihrer sinnlichen Gestalt zu unterschiedlichen Gebrauchsweisen aufzufordern (Verbeek 2005). Damit eröffnen sie unterschiedliche leibliche Zugänge zur Welt. Beispielsweise nutzen wir als kompetente Autofahrer nicht nur unser Fahrzeug, sondern gehen auch eine leibliche Beziehung zu ihm ein und erspüren seine Ausmaße beim Durchqueren einer Einfahrt, ohne diese Maße exakt benennen zu können (Merleau-Ponty 1976: 172f.). Die Welt erscheint aus Sicht eines solchen „embodied driver-car“ (Dant 2004: 71ff.) als System aus gangbaren und nicht gangbaren Wegstrecken, Verkehrshindernissen, anderen Verkehrsteilnehmern usw. Dinge gehen dabei nicht vollkommen im menschlichen Gebrauch auf, sondern entziehen sich mitunter dem menschlichen Zugriff (Waldenfels 2006): Sie gehen zu Bruch, quittieren den Dienst oder werfen Fragen hinsichtlich ihres angemessenen Gebrauchs auf. Auch in der Pädagogik weisen phänomenologische Autoren auf den vielstimmigen Aufforderungscharakter der Dinge hin (Langeveld 1955; Meyer-Drawe 1999; Stieve 2008). Das Interesse gilt hier den Kindern und ihrer kulturell noch wenig gerahmten Empfänglichkeit für die Aufforderung der Dinge, die erst im Laufe der Sozialisation einer konventionalisierten Gebrauchsweise weicht.

(3) Gebrauch: Andere Autoren suchen nach der praktischen Relationierung der Dinge. So will etwa die praxistheoretische Techniksoziologie (Hörning 2001) der Bedeutung der Dinge über den jeweiligen Gebrauch nachgehen: „Die Alltagsbedeutung eines technischen Dings, einer Sache, eines Sachverhalts steckt in den sozialen Praktiken", so Hörning (2005: 309). Ein technisches Artefakt stabilisiert dementsprechend nicht soziale Ordnung, sondern ist „Produzent und Provokateur von Unbestimmtheiten" (Hörning 2005: 308), denen der praktische Gebrauch begegnen muss. Die ethnomethodologischen Studies of Work (Garfinkel et al. 1981) bzw. die daran anschließenden Workplace Studies (Knoblauch \& Heath 2006) beschreiben Arbeit in all ihren profanen Details. Dabei geraten auch die Gerätschaften und Artefakte in den Blick, die zur Ausübung einer Tätigkeit gehören. Erst in der Interaktion erhalten die Dinge am Arbeitsplatz ihre (je vorläufige) Bedeutung (Hindmarsh \& Heath 2000: 558): „the sense and significance of objects emerge within the developing course of action and interaction; their objective and determinate sense is intersubjectively and momentarily accomplished ,here and now." 'In der qualitativen Bildungsforschung findet sich eine derartige Haltung bei Studien, die betonen, wie Dinge durch Gebrauch und Interaktion als pädagogische bzw. fachliche Objekte hervorgebracht werden (Bollig et al. 2012; Greiffenhagen 2014; Lynch \& Macbeth 1998; Wiesemann \& Lange 2014).

Wenn ich hier den schulischen Transformationsleistungen im Umgang mit den Dingen folge, so nehme ich eine Haltung ein, bei der die Dinge in erster Linie durch ihren praktischen Gebrauch einen Wandel erfahren. Damit soll für den Fall des mathematisch-naturwissenschaftlichen Schulunterrichts die Frage nach der spezifischen hier geleisteten Transformation beantwortet werden. Gleichzeitig treten auch Fragen des Arrangements und des körperlichen Bezugs auf. Schulische Dinge sind eingebunden in spezifischen Arrangements, die sich aus unterschiedlich zueinander konfigurierten Dingen und Menschen (etwa im Klassenzimmer) zusammensetzen. Zugleich sind die schulischen Dinge je spezifisch auf menschliche Körper mit ihren Sinnen bezogen und fordern diese $\mathrm{zu}$ unterschiedlichen Haltungen ihnen gegenüber auf.

\section{Die Transformation schulischer Dinge als praktische Arbeit}

Dinge durchlaufen in der Schule eine kulturelle „Biographie“ (Kopytoff 1986): Ihr Status und ihre Bedeutung sind nicht fixiert, sondern ändern sich entlang einer Abfolge von Phasen, die durch einen unterschiedlichen Umgang mit ihnen gekennzeichnet sind. Drei Phasen können für den lehrerzentrier- 
ten mathematisch-naturwissenschaftlichen Schulunterricht identifiziert werden (siehe Abb.1): Als Teil naturwissenschaftlicher Sammlungen sind die Dinge zunächst Teil eines curricular geordneten schulischen Archivs, aus dem sich die Lehrer die passenden Bestandteile eines Experiments zusammenstellen und damit zugleich ihren Unterricht antizipierend vorwegnehmen (3.1). Mit Unterrichtsbeginn vollziehen die Dinge einen Ortswechsel und gelangen von der Sammlung in den eigentlichen Unterrichtsraum. Hier müssen sie zu Beginn sprachlich und gestisch eingeführt werden. In der Durchführung der Experimente sind es dann durch einen Lehrer angeleitete Wahrnehmungsakte, die das Ding in einer fachlich relevanten Weise auftreten lassen (3.2). Der entscheidende Schritt findet nun mit einem medialen Wechsel statt, bei dem präsente Dinge in die sprachliche bzw. schriftliche Form übersetzt und dadurch re-präsentiert werden (3.3) (vgl. Mollenhauer 1991). Hier werden aus kontingenten Einzelfällen generalisierbare Beschreibungen und Aussagen gemacht (etwa eine ein Naturgesetz beschreibende Formel). Damit verändert sich auch der Status der so dargestellten Dinge, die nunmehr ihre lokale, vor der Klassenöffentlichkeit aufgeführte Transformationsgeschichte abgelegt haben. Schließlich zeigt sich auch, dass die Dinge selbst nach bestimmten didaktischen Kriterien gestaltet sind und sich so einer Objektivierung andienen (3.4).

\subsection{Ordnen und Vorbereiten: Dinge im Archiv}

Noch bevor der Unterricht beginnt, bereiten die Lehrpersonen ihre experimentellen Arrangements und Anschauungsobjekte auf den Unterricht vor. In den naturwissenschaftlichen Fächern stehen hierzu die sogenannten Sammlungen bereit, in denen sich in Regalen und Schränken ein Sammelsurium an Dingen („Experimente“) findet, mit denen naturwissenschaftliches Wissen an die Schüler vermittelt werden soll (siehe Abb.2). Die meisten Regale und Schränke in der Sammlung sind einzelnen curricularen Themen zugeordnet und weisen dies auch sichtbar durch entsprechende Beschriftungen aus (z. B. „Mechanik 1“, „Elektrostatik 2“).

Ortswechsel

\begin{tabular}{l} 
Sammlung \\
\hline Präsenz der Dinge \\
\hline (1) Dinge im Archiv
\end{tabular}

Medienwechsel

Abb.1 Drei „biographische" Phasen naturwissenschaftlicher Dinge

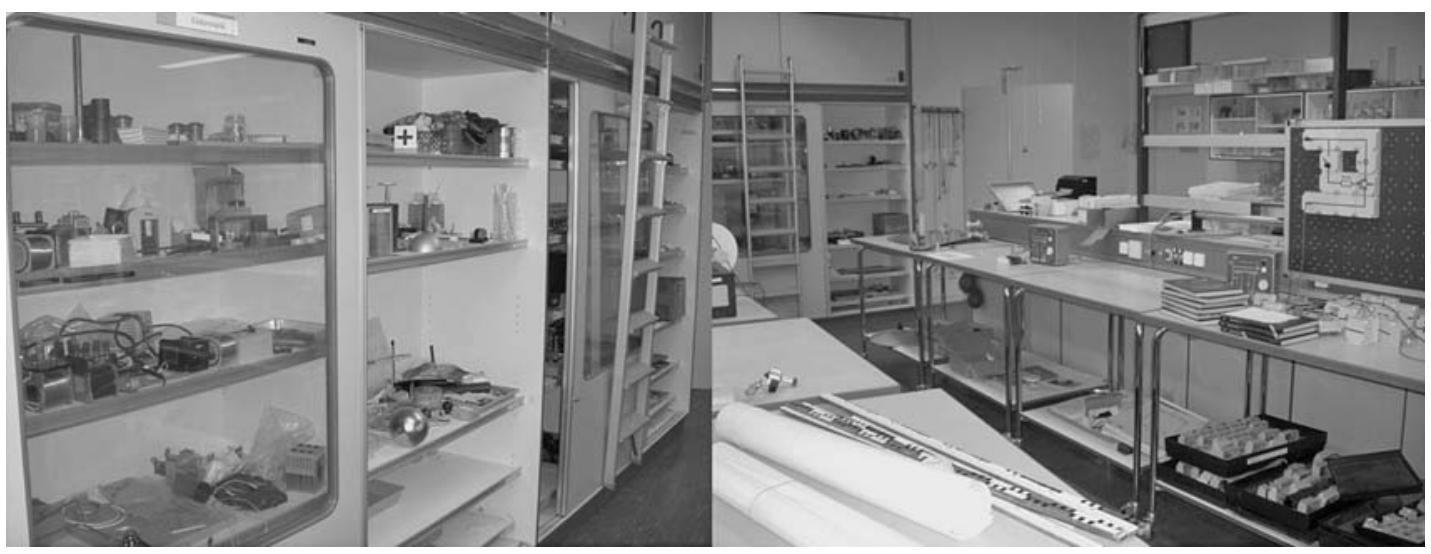

Abb. 2 Eine naturwissenschaftliche Sammlung 
Zur Unterrichtsvorbereitung greifen die Lehrkräfte gemäß dem Lehrplan ein thematisch passendes Experiment aus dieser Fülle an Dingen heraus. Oft müssen vorbereitend einzelne Bauteile miteinander verbunden und $\mathrm{zu}$ experimentellen Arrangements zusammengefügt werden. Hierzu greifen die Lehrer auf weitere Artefakte in der Sammlung zurück: Kabel, Messgeräte, Transformatoren, Ständer usw. Insbesondere Kabel und andere oft verwendete Bauteile sind auch dadurch von den thematisch geordneten Dingen unterschieden, dass für sie kein sie individualisierender und thematisch einordnender Platz vorgesehen ist. Sie werden nicht nach Themen, sondern entsprechend ihrer Funktion aufbewahrt; entweder in eigens dafür vorgesehenen und ebenfalls beschrifteten Schränken („Voltmeter") oder an nicht näher bestimmten Plätzen, an denen sie unterschiedslos über- und nebeneinander abgelegt werden. Neben der curricularen Ordnung kann man dementsprechend noch weitere Ordnungsprinzipien ausmachen: Auf den Tischen, aber auch innerhalb der Schränke bzw. auf den Regalen sind viele Dinge nach pragmatischen und ad hoc entstandenen Kriterien geordnet: häufig Benötigtes in Griffhöhe innerhalb der Schränke; Experimente, an denen man derzeit arbeitet, außerhalb der Schränke auf einer Arbeitsfläche; Gleiches zu Gleichem (z. B. an einem Haken hängende Stromkabel). Die Sammlung hat deshalb auch den Charakter einer abgeschlossenen Werkstatt (vgl. Willems 2007: 219). Hier können die Lehrer die Experimente ungestört und unbeobachtet vorbereiten. Dem Unterricht und seinen Zwängen sind sie dabei zeitlich und räumlich enthoben.

Die Klausur der Lehrer bei der Vorbereitung macht deutlich, dass es sich um sensible Vorgänge handelt. Mit der gelungenen Durchführung der Experimente vor der Klasse steht sowohl der reibungslose Ablauf der Unterrichtsinteraktion als auch die Darstellung als kompetenter Fachlehrer auf dem Spiel. ${ }^{1}$ Beim Zusammenstellen des Experiments geht es dementsprechend nicht nur darum, die als unwichtig erachtete Montage aus dem Unterricht herauszuhalten und so Zeit für das aus Lehrersicht eigentlich Relevante - die Vermittlung des „Stoffs“ - zu gewinnen. Es gilt vielmehr, in einer vorgreifenden „Probe“ (Goffman 1980: 72ff.) auch das mögliche Auftreten von Pannen und Fehlfunktionen zu ver-

\footnotetext{
${ }^{1}$ Ethnografische Studien zur Frühpädagogik (Schulz 2013; Neumann 2012) belegen in ähnlicher Weise, dass mit einem spezifischen Umgang mit Dingen pädagogisches Handeln überhaupt erst als solches hervorgebracht und darstellbar wird.
}

meiden. Hierzu testen die Lehrpersonen die Funktionsfähigkeit der Geräte, spielen den Versuchsablauf durch und überprüfen gemessene Werte auf ihre didaktische Plausibilität und Sichtbarkeit. Nur wenn sich die Messergebnisse konsistent mit den zu erwartenden Werten zeigen - wenn Messfehler also ausgeschlossen werden können - und sie in einem Bereich liegen, der auf der Skala deutlich zu sehen ist, können sie die Experimente beruhigt in den Unterricht mitnehmen. Einerseits geht es also darum, einen möglichst reibungslosen Ablauf zu sichern, andererseits steht aber auch die didaktische Sichtbarkeit eines Phänomens im Vordergrund: Ein Experiment muss vor einer Klasse sichtbar sein und sich mit dem schulisch zu vermittelnden „Stoff“ decken.

Oft finden Zusammenstellung und probende Durchführung auf Rollwagen statt, die einzelnen Lehrern zugeordnet sind (siehe Abb. 3). Die rund $1 \times 1 \mathrm{~m}$ breiten Rollwagen sind in zwei Ablageflächen unterteilt. Auf der oberen Fläche werden Versuchsaufbauten abgelegt, die in der nächsten Stunde unmittelbar Verwendung finden sollen. Die untere Fläche beheimatet hingegen neben Ersatzgeräten und -kabeln eine Reihe von Experimenten, die der Klasse bereits vertraut sind und bei Bedarf noch einmal gezeigt werden können. Der Rollwagen setzt damit auch eine zeitliche Ordnung des Unterrichtens um und unterteilt die darauf versammelten Dinge in mittelbar und unmittelbar Relevantes. Die Anordnung der Dinge symbolisiert also nicht bloß ein fachliches Wissen, sondern ist selbst

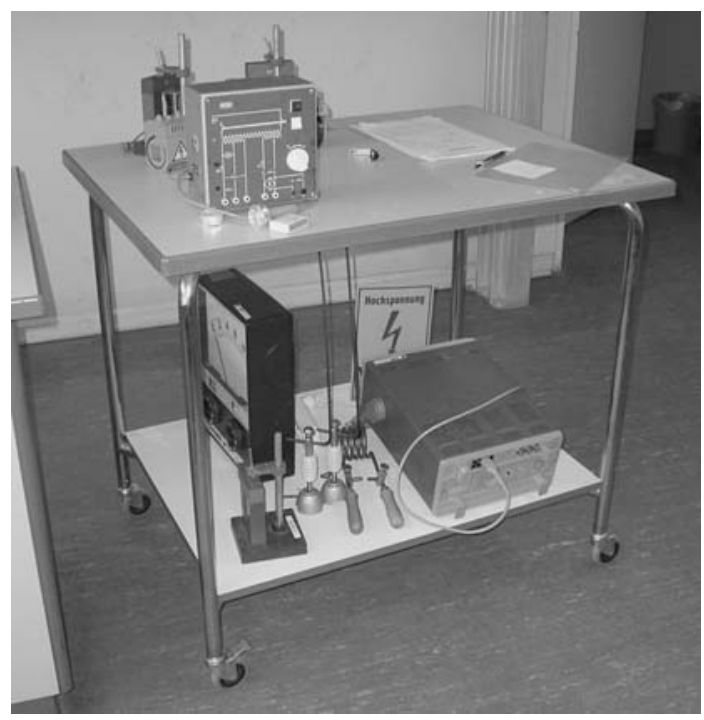

Abb. 3 Ein Rollwagen 
kognitives Werkzeug, in dem sie Handgriffe und Abläufe in Erinnerung ruft und strukturiert (vgl. Lynch et al. 1985: 199ff.). Mit den auf den Rollwagen angeordneten Dingen nehmen die Lehrkräfte eine Ordnung des Unterrichthaltens vorweg und versuchen, den Verlauf des Unterrichts vorab zu gestalten.

Mit ihren vorbereitenden Tätigkeiten in der Sammlung leisten die Lehrer zweierlei: Zum einen überführen sie die zirkuläre Ordnung des Curriculums in die (vergleichsweise) lineare Ordnung einer Unterrichtsstunde; zum anderen halten sie unerwünschte Pannen und Störungen vom Unterricht fern. Aus Sicht der Lehrperson darf es keine überraschende Erkenntnis geben, vielmehr soll kanonisches Wissen reproduziert werden. Auf ihrem Rollwagen eignen sie sich ein curriculares Themengebiet für die Dauer einer oder mehrerer Unterrichtsstunden an und bereiten die im Unterricht durchzuführenden Experimente so weit wie möglich vor.

In den Sammlungen überschneiden sich folglich mehrere aufeinander bezogene Ordnungen: ${ }^{2}$ (1) eine curriculare Ordnung thematisch bedeutsamer Experimente (Sammlung als Archiv); (2) eine pragmatische Ordnung der Vorbereitung und Zusammenstellung konkreter experimenteller Arrangements (Sammlung als Werkstatt); (3) die lineare Ordnung des Unterrichthaltens (Unterteilung des Rollwagens). Wenn die Dinge den Unterricht betreten, haben sie bereits didaktische Zurichtungen erfahren: als Bestandteil unterschiedlicher Ordnungen hinsichtlich ihrer Bedeutungsvielfalt sowie als zuverlässige technische Artefakte hinsichtlich ihrer materiellen Widerspenstigkeit und Unberechenbarkeit. Curricular verwahrte Gegenstände werden in später im Unterricht zu gebrauchende experimentelle Arrangements überführt. Die Dinge sind für die Lehrer dabei didaktische Werkzeuge, die für Themen und Unterrichtsepisoden stehen, in denen sich ein fachlich relevanter Sachverhalt deutlich zeigen soll. In der Vorbereitung ist die Lehrperson zugleich Bastler als auch vorausplanende Instanz ,ihres' Unterrichts. Die Schüler sind hingegen systematisch von der Vorbereitung ausgeschlossen.

\footnotetext{
2 Ähnliche Überschneidungen finden sich auch im Kontext musealer Aufbewahrungspraktiken (Doering \& Hirschauer 1997).
}

\subsection{Ein- und Durchführen: Dinge in Aktion}

Was passiert nun, wenn die Dinge die Sammlungen verlassen und in die Unterrichtsräume gelangen? Zum Unterrichtsbeginn tragen die Lehrpersonen die Versuchsaufbauten in die Fachräume oder schieben sie auf den Rollwagen dort hinein. Oft bleibt der Rollwagen fürs Erste etwas abseits neben dem Pult stehen. Er bzw. das darauf aufgebaute Experiment sind in die Peripherie des zentralen Pultbereichs verbannt und in der räumlichen Ordnung des Unterrichtsraums als (noch) nicht relevant gesetzt. Administrative Aufgaben (wie der Eintrag in das Klassenbuch) werden erledigt und letzte Handgriffe am Experiment vorgenommen: Kabel werden miteinander verbunden, Stecker eingesteckt etc. Durch halblaute Kommentare weisen die Lehrpersonen diese Handgriffe als vorbereitende Tätigkeit aus und signalisieren den Schülern, dass diese fachlich nicht relevant sind. Mal tun sie dies explizit („Das gehört noch nicht dazu!“), mal eher implizit: „So, jetzt müsste es gehen!“ - die vorherigen Handgriffe waren also nur die Ermöglichung dessen, worauf es ankommt.

Oft führen die Lehrer zunächst mündlich in ein Themengebiet (z.B. „Brechung des Lichts“) ein und leiten dann zum Experiment über. Dies geht häufig mit ostentativen „physical re-arrangements“ (Mehan 1979: 37) des Unterrichtsraums einher: Lehrpersonen schieben den Rollwagen Richtung Pult, stellen den Versuchsaufbau dort an zentraler Stelle ab oder setzen Schüler um. Dies führt nicht nur zu einer erhöhten Sichtbarkeit und räumlichen Prominenz der Dinge, sondern signalisiert als ,zeitliche Klammer" (Goffman 1980: 420ff.) auch deutlich einen neuen Abschnitt innerhalb des Unterrichtsgeschehens.

Als nächstes erhalten die Dinge für und vor der Klasse einen Namen und werden so auch sprachlich in das Unterrichtsgeschehen eingeführt. Die Lehrer zeigen dazu auf die Dinge oder halten sie ostentativ in die Höhe und benennen dabei, was zu sehen ist: „Ich hab euch ein Prisma mitgebracht!“ Oft bleibt es auch bei der einfachen Bestimmung als Experiment: „Hier ist ein kleines Experiment. Ich dreh' mal an dem Teil da." Manchmal sind auch die Schüler aufgefordert, den Namen für das Ding oder das sich daran zeigende Phänomen zu finden. Auch dann halten die Lehrer die Dinge meist demonstrativ vor sich in die Höhe und fragen beispielsweise „Was ist das eigentlich? “ Dies gleicht oft einem aussichtslosen Ratespiel, bei dem die Schüler die Antwort gar nicht kennen können. Die Lehrer weisen dann die erfolglosen ,Taufversuche‘ der Schüler zu- 
rück: „Kein Föhn oder eine Weihnachtsröhre. Das Ding hat einen Namen: Elektronenstrahlablenkröhre. Ganz nüchtern. “ Der Lehrer kann in diesem Fall als ein Experte auftreten, der mit dem Namen des Dings auch die dahinterliegende Physik kennt. Anders als die Alltagsbegriffe der Schüler kennzeichnet der fachliche Begriff die „nüchterne“ Welt der Physik und weist dem Bauteil eine physikalisch bestimmte Funktion zu.

Neben einer globalen Benennung des Arrangements greifen die Lehrpersonen in einigen Fällen einzelne Bestandteile eines experimentellen Arrangements heraus und benennen sie individuell:

Nach einer mündlichen Einführung zum Thema „Lichtbrechung“ verkündet Herr Martin: „So, ein kleines Experiment!" Der Lehrer stellt sich neben den Rollwagen, zeigt auf den Versuchsaufbau und erläutert: „Ich habe hier ein Gefäß gefüllt mit Wasser, eine Centmünze und ein Kupferrohr.“

(Physik, 7. Klasse)

Auffällig ist, dass nicht alle Bestandteile einen $\mathrm{Na}$ men erhalten. Im obigen Beispiel finden etwa eine Halterung und Schrauben, aber auch der Rollwagen, auf dem das Experiment abgestellt ist, keine explizite Erwähnung. Mit der gestisch unterstützten Benennung der Dinge markieren und selektieren die Lehrer einzelne Bestandteile und heben sie so hervor. Manchmal drängen sich aber einzelne fachlich irrelevante Dinge derart auf, dass sie ausdrücklich zu irrelevanten Hilfsmitteln erklärt werden müssen:

Dann nimmt Herr Baier einen Holzkasten vom Rollwagen, stellt diesen auf das Pult und versetzt darauf die Stimmgabel in Schwingung. Der Kasten, so erläutert er, sei „nur dazu da, als Resonanzkörper zu dienen“.

(Physik, 10. Klasse)

Hieran wird abermals deutlich, dass die prominente Platzierung von Dingen auf dem Pult aus der Sicht der Unterrichtsteilnehmer einen symbolisch äußerst aufgeladenen Akt darstellt. Dementsprechend muss der Holzkasten sprachlich deklassiert werden, damit sich die Aufmerksamkeit auf das eigentlich relevante Phänomen (hier: ein von einer Stimmgabel erzeugter Ton) richten kann. Dies gilt nicht für alle auf das Pult gestellten Gegenstände gleichermaßen. Wenn etwa die Lehrer zu Beginn einer Schulstunde ihre Taschen, Schlüssel und Stifte auf dem Pult ablegen, ist dies gegenüber den Schülern nicht erklärungsbedürftig - diese Gegenstände sind ihnen vertraute Mitbringsel der Lehrperson. Erst wenn unvertraute oder schlicht schulisch nicht-alltägliche Dinge auf dem Pult platziert werden, wirft dies Fragen auf. Platzierung und (schulische) Vorgeschichte der Dinge gehen eine Wechselwirkung ein, die gegebenenfalls Handlungsbedarf erzeugt.
Wurden die Dinge sprachlich als Objekte von Interesse markiert und mit einem Namen versehen, kann die eigentliche Durchführung des Experiments beginnen. Hierzu zeigen die Lehrer ihren Schülern mit kontextualisierenden Markern (z. B. „so“, „gut“, „okay“; vgl. Dorr-Bremme 1990) an, dass ein neuer Abschnitt beginnt, und geben zugleich einen geeigneten Zeitpunkt für die Beobachtung des Versuchs vor: „So, also.“; „Also, okay, auf die Plätze, fertig, los!" Gerade Experimente im Bereich „Mechanik“ sind Ereignisse, denen es im richtigen Moment beizuwohnen gilt, wollen die Schüler nichts verpassen. Mehr noch: Die Lehrer bringen durch ihre zeitlichen und kontextualisierenden Hinweise ein physikalisch relevantes Ereignis überhaupt erst hervor, denn oftmals ist den Schülern nicht einsichtig, was es zu beobachten gilt. Bleiben derartige Hinweise aus, fragen die Schüler bisweilen, ob der leichte Ausschlag eines Zeigers oder ein subtiler brummender Ton schon zum Experiment dazugehören.

Zusammen mit dem ,Startschuss' geben die Lehrer oft angemessene Rezeptionsweisen vor: „Alle nach vorne schauen!"; „So, wenn ihr jetzt mal hinschaut!“ „Stifte aus der Hand, Heft zu! Jetzt gibt's noch was zu gucken.“ „Stifte liegen lassen! Schaut her!" Damit ist der übliche modus operandi des Schulunterrichts außer Kraft gesetzt: Stifte und Hefte sollen außen vor bleiben; das fixierende und reproduzierende Abschreiben eines autorisierten Wissens soll zugunsten eines schauenden Bezeugens eines Ereignisses in den Hintergrund treten. Dies stellen die Lehrpersonen auch am eigenen Körper dar: etwa indem sie aufhören, zur Klasse zu sprechen, ihren Körper von den Schülern abwenden und zum Experiment ausrichten, ihre Stirn in Falten legen und mit zusammengekniffenen Augen auf das Experiment schauen.

Experimentelle Arrangements und Schüler treffen durch die typische Anordnung der Klassenzimmer in großer Distanz aufeinander. Die Schüler sind an ihren Tischen und Stühlen auf ihren Oberkörper reduziert (vgl. Hnilica 2010). Sie sollen dem Geschehen schreibend, zuhörend, sehend und sprechend beiwohnen. Diese sinnlich-praktische Reduktion zielt auf eine spezifische Rezeptionsweise. Der vorgängige Sinn, an den die Lehrer appellieren, ist der distanzierte Sehsinn. Dieser gilt der Schulphysik als der primäre Erkenntnissinn. Wenn man die Unterrichtsteilnehmer dabei beobachtet, wie sie dieses Sehen handhaben, wird deutlich, dass es sich dabei um ein Wahrnehmen unter Anleitung handelt. Die Lehrer richten den Sehsinn der Schüler durch ihre Anweisungen und Gesten gezielt aus. Sie lenken den Blick der Schüler beispielsweise dadurch auf 
physikalisch relevante Vorgänge, dass sie einzelne Phänomene benennen oder aber gezielt zu irrelevanten Bestandteilen erklären:

Bei einem Experiment zur Lichtbrechung zeigt Herr Martin auf einen abgelenkten Lichtstrahl: „Seht ihr das hier? Das, was ihr hier seht ((zeigt auf die Reflexion des Plexiglases)), ist eine normale Reflexion. Das Plexiglas reflektiert leider, darum geht es aber nicht!“ (Physik, 7. Klasse)

Die Lehrperson bezeichnet hier sinnlich erfahrbare Phänomene („Reflexion“) mit den Fachbegriffen und scheidet dabei Relevantes vom Irrelevanten („darum geht es aber nicht!“). Sie arbeitet damit an einer selektiven Wahrnehmung des Experiments. Ohne diese Hinweise und Tätigkeiten sehen die Schüler an den Experimenten und Anschauungsobjekten nichts von Belang: Sie sehen dann statt des Schlauchs eines Gebläses, der zusammen mit einem Tischtennisball das Kräftegleichgewicht veranschaulichen soll, einen „Staubsauger“ und teilen dies lautstark der Klasse mit.

Die Arbeit an der Wahrnehmung wird dann besonders deutlich, wenn ein Versuch nicht wie geplant abläuft oder partout nicht gelingen will. Die Lehrer erklären dann kurzerhand, was „eigentlich“ zu sehen sein sollte, markieren mit ihren Fingern die eigentliche Nullstellung einer Messung oder greifen beherzt korrigierend zu, wenn ein Stapel Kopierpapier sich nicht so verhält wie er soll: „Das soll jetzt nicht schlapp herunterhängen ((biegt die Ecke hoch))." Sie sind entweder gezwungen, die sinnliche Erfahrung des Experiments in das Medium der Sprache zu übersetzen oder müssen manipulativ eingreifen.

Die Unterrichtsteilnehmer arbeiten bei der Ein- und Durchführung der Experimente daran, die experimentellen Dinge in den Vordergrund zu stellen. An und mit den Dingen soll sich etwas zeigen: Lichtstrahlen und ihre Reflektionen, als Töne wahrnehmbare Schwingungen usw. Sich selbst nehmen die Lehrpersonen hingegen zurück. Sie gerieren sich als Vorführer eines Spektakels, dem es in je bestimmter Rezeptionshaltung (geleitetes Sehen oder Hören) zu folgen gilt. Aus für einen antizipierten Unterricht vorbereiteten experimentellen Arrangements werden tatsächlich durchgeführte und unter Anleitung wahrgenommene Experimente - aus dem konjunktivischen Modus der Vorbereitung („So müssten sie es sehen!") wird ein imperativer Modus der Durchführung („Guckt genau hin!“). Damit erhalten die Dinge den Status von wissensvermittelnden Instanzen. In diesem Moment delegiert die Lehrperson Handlungsträgerschaft teilweise an die Dinge (vgl. Latour 2001): Statt selbst etwas zu er- läutern, ,sprechen' gewissermaßen für einen kurzen Augenblick die Dinge. Die Auswirkungen dieser Transformation kann man unmittelbar an den Schülern beobachten. Schüler stellen zum Zeitpunkt der Durchführung die Gespräche untereinander ein, lehnen sich bisweilen mit dem gesamten Oberkörper weit nach vorne oder stehen gar auf, um dem Geschehen folgen zu können. Diese enge leibliche Einbindung der Schüler drückt sich besonders dann aus, wenn ein Experiment trotz Ankündigung nicht durchgeführt werden kann. Die Schüler zeigen sich dann enttäuscht („Ach Mann!“), versprechen die Experimente doch Abwechslung vom „interaktionalen Gleichfluss“ (Krummheuer 2002: 46) des Schulunterrichts mit seiner schematischen Abfolge von Fragen, Antworten und Kommentaren (hierzu Mehan 1979). Wenn das Experiment dann doch noch durchgeführt werden kann, stimmen die Schüler in der Mittelstufe schon einmal einen freudigen Halleluja-Gesang an. Dennoch bleibt diese den Dingen zugeschriebene Wirkmacht eingeschränkt. Sie ist durch sprachliche und gestische Hinweise gerahmt und zudem zeitlich begrenzt.

\section{3 Übersetzen: Dinge in Wort und Schrift}

Bereits mit dem Benennen einzelner Bestandteile des experimentellen Arrangements beginnen die Lehrpersonen damit, die Dinge in sprachliche Form zu überführen und sie somit medial zu re-präsentieren. Statt der dinglichen Verkörperung eines naturwissenschaftlichen Phänomens, finden sich dann verbalsprachliche und schriftliche Zeichen, die mittelbar auf das Experiment verweisen. Dieser für den naturwissenschaftlichen Unterricht zentrale Übersetzungsvorgang setzt sich nach dem Experiment fort. Nun sind die Schüler gefragt, das Gesehene zu beschreiben:

Mit dem Modell einer Pyramide in der Hand bittet Herr Frankfurter die Schüler: „Kann jemand die Pyramide beschreiben, wie wir das Prisma beschrieben haben?" Die Schüler versuchen es und scheitern an der mathematischen Sprache. Herr Frankfurter mahnt: „Gewöhnt euch eine genaue Sprechweise an!“ Während die Schüler versuchen, die Pyramide mathematisch genau zu beschreiben, hat er die Pyramide die ganze Zeit in der Hand und zeigt immer wieder einzelne Strecken und Flächen an, die gerade von den Schülern genannt werden. (Mathematik, 10. Klasse)

Das Einfordern einer „genauen Sprechweise“ führt die Schüler in das Sprachspiel einer schulischen Disziplin ein. Der Aufruf des entsprechenden sprachlichen Registers geschieht hier durch den Verweis auf ein bereits gezeigtes und adäquat beschriebenes schulisches Artefakt. Im Sprechen über die Dinge 
versichern sich die Unterrichtsteilnehmer einer hinreichend geteilten Sicht auf die Dinge und schaffen ein „interindividual object“ (Hindmarsh \& Heath 2000: 555), auf das im Lehrgespräch Bezug genommen werden kann. Ein Objekt wird hervorgehoben, diskursiv gerahmt und fachlich eingeordnet. Insofern kommt der Zeigegeste des Lehrers eine entscheidende Rolle zu. Mit ihr verknüpft der Lehrer immer wieder Dinge und die an ihnen sich zeigenden Phänomene mit ihrem sprachlichen Ausdruck (vgl. Bauersfeld 1995). Die Zeigegeste ist das Bindeglied zwischen dem konkreten Ding und dem abstrakten sprachlichen Ausdruck. Oftmals ersetzen die Lehrer dabei ganz explizit unpassende durch passende Begriffe. Als etwa die Schüler in einem Versuch zur Lichtbrechung das Verhalten von Lichtstrahlen beschreiben sollen, verwenden sie Begriffe wie „hochgehen“ und „runtergehen“, woraufhin sie ihr Lehrer korrigiert: „Es heißt reflektieren und brechen! " Der Verweis auf den korrekten Ausdruck ist dabei keinesfalls eine einseitige Übertragung vom Ding bzw. dem daran sichtbar werdenden Phänomen in das Medium der Sprache. Durch die mediale Übertragung erfahren auch die dargestellten Dinge eine Veränderung: Sie stehen nun nicht mehr nur für sich selbst, sondern für eine ganze Gattung von Objekten (etwa für Pyramiden oder Prismen) und Phänomenen sowie für die dazugehörige Formel. Mit dem Hinweis auf das bereits gezeigte Ding helfen die Lehrer ihren Schülern oftmals in dieser Richtung auf die Sprünge. Zum Nutzen eines geometrischen Modells äußert sich ein Lehrer dementsprechend:

Und da kann man's einfach sehen und da hab ich so'n bisschen die Hoffnung, dass man dann einfach sagen kann: „Aber ich hab euch doch da die Pyramiden mitgebracht." Das war sogar in einer Stunde mal, als ich die Formel abhören wollte und sie's nicht wussten und dann konnte ich sagen: „Ich hab euch doch extra das mitgebracht!“ „Ach so, ja! Ein Drittel, ne?“

(Interview mit einem Mathematiklehrer)

Nach der mündlichen Versprachlichung folgt die Verschriftlichung der beobachteten Experimente. Träger dieser Verschriftlichung ist zunächst die klassenöffentliche Wandtafel. An ihr notieren die Unterrichtsteilnehmer Skizzen des Experiments und fixieren Beobachtungen als Aussagesatz („Beobachtung: Bei gleichzeitigem Start treffen beide Kugeln gleichzeitig auf.“), visualisieren Zusammenhänge in Form eines Diagramms oder halten allgemeingültige Formeln fest. Auffällig ist dabei, dass die praktischen Bemühungen der Unterrichtsteilnehmer, aber auch kontingente Ereignisse und zahlreiche Details keine Erwähnung mehr finden:
Nachdem er mit den Schülern einige Alltagsbeispiele zum Thema „Waagrechter Wurf“ durchgegangen ist, wendet sich Herr Grabow dem auf dem Pult aufgebauten Versuch zu und kommentiert: „Also, Prinzip waagrechter Wurf wäre so was ((geht zu Versuchsaufbau und schraubt daran herum)). Also wir haben hier so 'ne Feder, die kann man spannen ((spannt sie)) und dann kann ich sie losschnackseln lassen und dann schießt sie halt irgendwo hin ((lässt die Feder schnappen)). Fantastisch, oder? “

Herr Grabow fährt fort: „So also, da kann man jetzt eben ein Kügelchen da draufsetzen. Und Vermutung, wie dürfte hier die Bahnkurve aussehen? Also so wie, gell, weiß ja jeder, ich mein Zeichentrick, hat ja auch jeder gesehen, des geht immer so ((macht mit den Händen vor, wie eine Zeichentrickfigur über einen Abgrund läuft)) und dann gucken sie, ,Ah, da is' ja der Boden!' ((macht abrupten Absturz pantomimisch vor, vereinzelt lachen die Schüler)) So sieht's immer aus.“

„Schauen wir, ob das auch im Realfall so is'. Oder ob da die lieben Zeichner doch immer etwas ein bisschen falsch gesehen haben. Also, okay, auf die Plätze, fertig, los ((betätigt den Auslöser))! “ Die Kugel fällt in einer Bogenbahn auf den Tisch, rollt zu Boden und springt dort zwei oder dreimal auf. Der Lehrer kommentiert: „Fantastisch. Also in etwa, kann man sich vorstellen. Kann den etwa einer noch mal beschreiben, wie sah denn das etwa aus? Diese Bewegung (kurze Pause) Petra." Petra weiß es nicht und auch die anderen Schüler geben keine Antwort. Herr Grabow sammelt die Kugel wieder auf und setzt sie abermals in die Apparatur ein: „Also noch mal, wir spannen vor ((spannt vor)), um noch einmal einen Blick auf den fantastischen Flug. Auf die Plätze, fertig, los ((betätigt Auslöser, Kugel fällt auch jetzt wieder auf den Boden und springt dort auf))!“ Nun will Herr Grabow wissen: „So, is' natürlich klar, wenn man des so nachmalen wollte, in etwa, Tim, wie sieht's aus?“ Tim: „So ((vollführt mit dem Zeigefinger eine Bogenbahn)). “ Der Lehrer greift die Geste auf und wiederholt sie gut sichtbar für die Klasse. Dann hält Herr Grabow diese gestisch dargestellte Bogenbahn in Form eines Diagramms an der Tafel fest.

(Physik, 11. Klasse)

Systematisch blenden die Unterrichtsteilnehmer im obigen Beispiel fachlich unwichtige Details aus (siehe Abb. 4): die mündliche Einführung des Experiments, der scherzhafte Bezug auf den Trickfilm, die wiederholte Durchführung und die daran anschließende Präzisierung der Frage hin zu einer visuellen Darstellung der Flugbahn („nachmalen“), aber auch Informationen zum Material, zur Farbe oder genauen Gestalt der Abschussapparatur oder eine Beschreibung dessen, was der Kugel widerfährt, nachdem sie auf dem Pult auftrifft. Der Lehrer bestätigt durch die Wiederholung der Schülergeste vor der Klasse, dass es hier einzig um einen idealisierten Ausschnitt der Flugbahn geht. Durch die sich anschließende Überführung in ein anderes, weitaus beständigeres Medium wird diese Haltung 


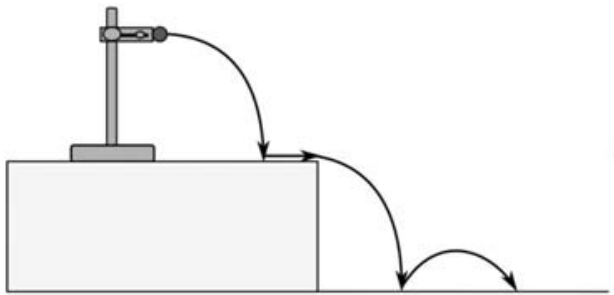

1

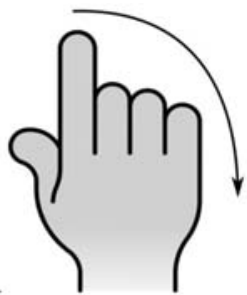

2

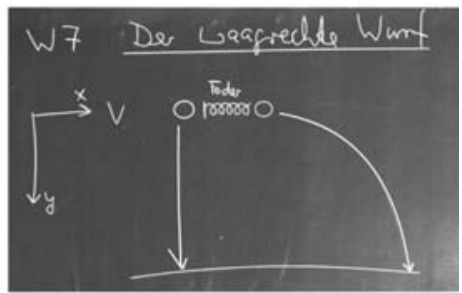

3

Abb. 4 Selektion relevanter Merkmale in der Verschriftlichung

weiter gestärkt: Einzig eine idealisierte Darstellung der Flugbahn und der Abschussapparatur bzw. des eigentlich relevanten, aber nicht unmittelbar sichtbaren Mechanismus („Feder“) sind dort zu sehen.

Liegen der Versuch und die dazugehörigen Zeichnungen, Diagramme, Merksätze, Formeln etc. in schriftlicher Form an der Wandtafel vor, können die Unterrichtsteilnehmer immer wieder darauf Bezug nehmen. Die Schüler halten das an der Wandtafel Angeschriebene in ihren Heften fest und erhalten so eine transportable Version des klassenkollektiven Wissens. Die ,Gesprächsmaschinerie des Schulunterrichts beruht auf der Annahme, dass alle Schüler über dieses Wissen verfügen (Kalthoff 1997: 91f.). Folglich können die Unterrichtsteilnehmer in späteren Schulstunden mit der Schrift so über das Experiment sprechen, als ob alle das Gleiche gesehen hätten: „Und wir haben gesehen, dass...", „Ihr habt gesehen, die passen hier alle in den Würfel rein!“ Dabei nehmen die Unterrichtsteilnehmer mit Hilfe der Zeichen an der Wandtafel auf das vergangene experimentelle Arrangement Bezug. So lässt etwa der Lehrer in der Unterrichtsstunde nach dem Experiment zum waagrechten Wurf zunächst die Formeln und das Diagramm der letzten Stunde wiederholen, um dann auf das Angeschriebene zeigend über den Flug der Kugel zu sprechen: „Und dann is' sie hier ((zeigt auf Bogenbahn)) so langgeflogen. “Zweierlei fällt dabei ins Auge:

(1) Die Zeichen ersetzen die einst präsenten Dinge und stehen für sie; die Unterrichtsteilnehmer ,greifen' durch sie hindurch auf das Experiment zu. Es herrscht ein semiotischer Realismus vor, der den Zeichen unterstellt, die Dinge gleichwertig zu ersetzen. Die Unterrichtsteilnehmer sprechen mittels der Zeichen über die Dinge.

(2) Es geht nicht darum, den Versuch in all seinen kontingenten Einzelheiten zu wiederholen, sondern als idealisierte und mathematisierbare
Form an der Tafel. Scheiden schon die Zeigegesten und indexikalischen Äußerungen der Lehrpersonen relevante von irrelevanten Elementen eines experimentellen Arrangements, so erlaubt es die Schrift, die aus schulischer Sicht unerheblichen Bestandteile vollends dem Vergessen anheimfallen zu lassen. Weder die Zwischenrufe und falschen Antworten der Schüler noch die Pannen und Missgeschicke im Umgang mit dem Versuchsaufbau tauchen hier auf.

Die Verschriftlichung in Form von Diagrammen, Merksätzen und Formeln bildet den Endpunkt einer schrittweisen Transformation präsenter Dinge. Dies stellt für die Wissenspraxis des mathematisch-naturwissenschaftlichen Schulunterrichts einen zentralen Vorgang dar, denn mit der Beherrschung der Transformation lernen die Schüler, Dinge fachlich wahrzunehmen und zu benennen. Im Mittelpunkt steht die Ermöglichung einer disziplinären Sicht auf die Dinge. Aus Sicht der Disziplin kontingente und idiosynkratische Elemente werden systematisch ausgeblendet. Die disziplinäre Sicht ist in zweifacher Hinsicht voraussetzungsvoll und beinhaltet mehr als die Aufnahme äußerlicher Sinneseindrücke: Erstens baut sie auf einem kognitiv-konzeptionellen Gerüst auf, das bestimmt, welche Schlüsse wir aus unseren Beobachtungen ziehen (Eberbach \& Crowley 2009; Fodor 1984); hier besteht dieses Gerüst vor allem aus den Kategorien der Schulmathematik bzw. -physik, das unter anderem durch sprachliche Ermahnungen bei den Schülern wachgerufen wird bzw. werden soll. Dementsprechend kann man zweitens die disziplinäre Sicht als Ergebnis einer Reihe von Praktiken verstehen (Goodwin 1994; Lynch \& Macbeth 1998; Schindler 2009). Im vorliegenden Fall zielen die praktischen Bemühungen der Unterrichtsteilnehmer vor allem auf die Dinge bzw. ihre Beziehung zu den Schülern: vorbereitende und ordnende Tätigkeiten innerhalb der 
Sammlung, ostentative Präsentation und Arrangement im Unterrichtsraum, selektierende Zeigegesten und Benennungen, Markierung geeigneter Beobachtungszeitpunkte und Rezeptionsweisen bei der Durchführung, idealisierende und verallgemeinernde Fixierung in Form schriftlicher und graphischer Zeichen. Am Ende der Transformationsleistung steht dann ein relativ klar umrissenes mathematisches oder physikalisches Objekt, das als unabhängig von den praktischen Bemühungen der Unterrichtsteilnehmer gilt.

Die Schüler und Lehrer wechseln in dieser Phase nicht nur das Medium der Darstellung (vom präsenten Ding zu seiner Re-Präsentation), sondern auch ihre jeweilige Haltung gegenüber dem Experiment. Die Lehrperson übernimmt nun wieder die Instruktion der Klasse und tritt durch ihre Anweisungen in den Vordergrund. Statt Vorführer eines Spektakels ist sie jetzt jemand, der um das ,Geheimnis' der dinglich dargestellten Phänomene weiß, sie mittels mündlicher Rede, Tafel und Kreide in die sprachliche Form übersetzen und dadurch generalisierende Erklärungen geben kann. Die Schüler wandeln sich hingegen von zunächst punktuell aufmerksamen Zuschauern eines Spektakels zu abschreibenden Reproduzenten eines durch die Lehrer autorisierten Wissens.

\subsection{Reduktion: Didaktische Gestaltung}

In ihren objektivierenden Bemühungen können die Unterrichtsteilnehmer auf eine spezifisch didaktische Gestaltung vieler schulischer Dinge aufbauen. Eine ganze „Lehrmittelindustrie“ (so die Eigenbezeichnung des Feldes) stellt verschiedene Gegenstände her, mit denen im Unterricht auf anschauliche Art und Weise schulisches Wissen vermittelt werden soll. Ein Beispiel hierfür sind die sogenannten Demonstrationsmessgeräte für den Physikunterricht (siehe Abb. 5). Betrachtet man diese, so fällt auf, dass vor allem zwei Prinzipien ihre Gestaltung bestimmen. Zum einen ist gegenüber herkömmlichen Messgeräten die Anzeige stark vergrößert: Auch Schüler in den letzten Reihen des Klassenzimmers können die Anzeige ohne Mühen erkennen. Zum anderen setzen die Demonstrationsmessgeräte neben der Vergrößerung auf eine didaktische Vereindeutigung. Beim abgebildeten Gerät handelt es sich um ein Multimeter, das verschiedene physikalische Größen (Stromstärke, Spannung und Widerstand) messen kann. Gleichwohl zeigt es stets nur den Messwert für eine dieser Größen an, die Anzahl der Anschlüsse und Schalter sind auf ein Minimum begrenzt. Die derzeit gemessene Größe und weitere

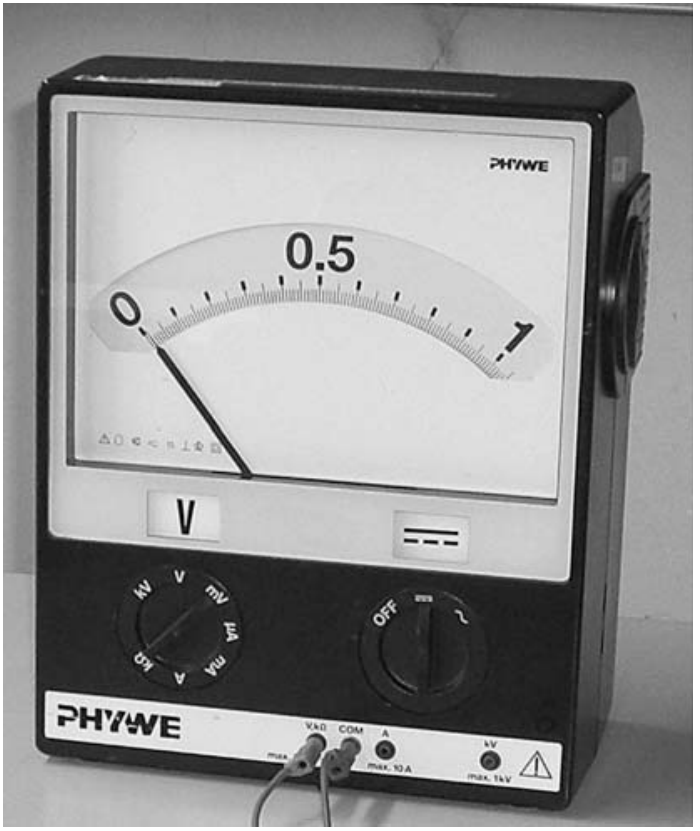

Abb. 5 Ein Demonstrationsmessgerät

Einstellungen sind - für den initiierten Schüler deutlich abzulesen; in diesem Fall: Spannung in Volt sowie Gleichstrom. Hinzu kommt, dass bei den meisten Geräten auf eine analoge Anzeige zurückgegriffen wird, obwohl digitale Anzeigen bei Messgeräten mittlerweile der Regelfall sind. Der analoge Zeiger ist ein klassischer „Index“ (Peirce 1983). Gegenüber der digitalen Anzeige bietet er einen entscheidenden Vorteil: Jede Veränderung des Messwerts hat nicht nur eine Veränderung eines Zeichens, sondern zugleich eine deutlich sichtbare Bewegung des Zeigers zur Folge. Schließlich weisen die Demonstrationsmessgeräte eine deutlich sichtbare Außen- und Vorderseite auf: Nur die außen liegende Vorderseite des Geräts weist in Richtung der Klasse und ist für sie sichtbar. Rückseite und Innenleben des Geräts sind den Schülern verborgen. Für den Schulunterricht sind sie irrelevante Blackbox; die Gestaltung des Geräts stellt den gut ablesbaren Messwert deutlich in den Mittelpunkt. Sie sind nicht bloß Messgeräte, sondern Demonstrationsmessgeräte vor einer und für eine Klasse.

Die Geräte sind also darauf ausgelegt, etwas vor einer Klassenöffentlichkeit deutlich und möglichst eindeutig zur Darstellung zu bringen. Ferner ist die Überführung in Zeichenform im Fall der Demonstrationsmessgeräte schon angelegt. Die Skala legt einen messenden Zugang zur Welt nahe und gibt einen genau bestimmbaren numerischen Wert an. Be- 
reits in der Lehrmittelindustrie wird so eine Form des Unterrichthaltens vorweggenommen, in der eine bestimmte Sicht auf die Dinge ermöglicht werden soll. Weder Farbe oder Größe noch Rück- oder Unterseite des Demonstrationsmessgeräts sind von Interesse, sondern einzig und allein die Messung eines Wertes. Die Unterrichtsteilnehmer können bei der Objektivierung auf diese didaktische Reduktion der Lehrmittel zurückgreifen. Dementsprechend gelten sie als „Rückgrat des Unterrichts“ (Oelkers 2010). Sie nehmen Lehrern und Schülern Arbeit ab, insofern ein Teil der Transformation der Dinge schon an anderer Stelle, nämlich in der Lehrmittelindustrie, vollbracht wurde.

Im Kontrast mit einem anderen Typus schulischer Dinge wird diese Leistung der Lehrmittelindustrie besonders deutlich. Neben eigens für didaktische Zwecke gefertigten Dingen greifen Lehrer auch auf andere Gegenstände zurück, um den Schülern mathematische und naturwissenschaftliche Zusammenhänge näherzubringen: herunterfallende Stifte, die den freien Fall visualisieren; Flugzeugmodelle als Beispiel für die Berechnung geometrischer Körper; Erbsen und Lautsprecher, die akustische Schwingungen sichtbar machen etc. Der schulische Einsatz derartiger Gegenstände erscheint gleichwohl prekärer und erfordert ein höheres Maß an situativer Arbeit an den Dingen: Die Gefahr ist groß, dass die Schüler den Dingen aus Lehrersicht nicht angemessen begegnen. Als etwa ein Lehrer mit einem Bündel Kopierpapier das Klassenzimmer betritt, reagiert eine Schülerin entsetzt: „Schreiben wir etwa einen Test? “ Erst mit seiner Entgegnung, dass er damit etwas zeigen wolle, kann er die Situation klären. Das Kopierpapier dient dazu, einen geometrischen Sachverhalt zu veranschaulichen („Das Cavalierische Prinzip“).

Andererseits kann aber gerade die Vieldeutigkeit nicht-didaktischer Gegenstände für didaktische Zwecke genutzt werden. An ihnen lässt sich einüben, was es heißt, die Welt aus Sicht einer schulischen Disziplin zu betrachten. Als etwa ein Mathematiklehrer einmal das Modell eines Flugzeugs mitbringt, kann er mit der Klasse erarbeiten, dass man das Volumen der Passagierkabine näherungsweise mit der Formel zur Berechnung des Volumens eines Zylinders berechnen kann. Gleichwohl müssen die Lehrer und Schüler lange an dieser didaktischen bzw. mathematischen Transformation des Flugzeugmodells arbeiten: Zunächst dient das Modell als Gesprächsanlass, um über die Passagierluftfahrt im Allgemeinen zu sprechen und um darauf aufbauend zum Problem des Luftdrucks in der Passagierkabine zu gelangen. Hieran schließt eine tech- nische Diskussion zu Geräten zur Luftaufbereitung an. Ein fiktives praktisches Problem wird ersonnen: Welches Gerät muss eine Fluggesellschaft für den durch das Modell dargestellten Flugzeugtyp kaufen? Schließlich wird daraus ein mathematisches Problem abgeleitet: Wie kann man näherungsweise das Volumen des Flugzeugs bestimmen? Schüler und Lehrer umkreisen das Modell diskursiv, bis sie schließlich bei einem mathematischen Problem anlangen. Das Flugzeugmodell bietet mit all seinen ikonischen Details einen Sinnüberschuss, der vom mathematischen Problem ablenkt. Immer wieder bringen die Schüler deshalb auch andere Aspekte in die Diskussion ein (Leistung der Motoren, Farbe des Flugzeugs etc.). Dem wirkt das Unterrichtsgespräch schrittweise entgegen, indem gemeinsam an einer mathematischen Sichtweise auf das Objekt gearbeitet wird.

Hier zeigt sich deutlich, dass die Gestaltung der Dinge an der Transformationsleistung beteiligt ist. Didaktisch gestaltete Artefakte offerieren die mühelose Einbindung in die objektivierende Transformation und bieten den zeigenden Praktiken der Lehrpersonen kaum Widerstand. Wenn die Schüler auch nicht immer die Mathematik oder Physik hinter den darstellenden Dingen begreifen, so verstehen sie doch zumindest deren didaktische Verwendung als fachliches Wissen repräsentierende Objekte. Ihre Leistung besteht deshalb darin, sich einem fachlichen Sehen anzudienen. Oftmals (etwa im Fall der Demonstrationsmessgeräte) legen sie die Überführung materiell sichtbarer Dinge und Vorgänge in Zeichenform schon dadurch nahe, dass sich an ihnen messbare Werte ablesen lassen. Ganz anders hingegen die nicht-schulischen Artefakte: Sie rufen bisweilen unpassende Assoziationen bei den Schülern wach, und es bedarf größerer Anstrengungen, um sie in fachliche Objekte zu transformieren. Gerade hieran können die Schüler aber ein fachliches Sehen erlernen, das sich an der Vieldeutigkeit des alltäglichen Artefakts schärft. Mit anderen Worten: Die situative Arbeit an derlei, wilden' Dingen kann auch als Ressource der schulischen Wissensvermittlung dienen. Schüler lernen dann, was es heißt, die Welt physikalisch oder mathematisch zu betrachten.

Die didaktische Gestaltung der Lehrmittel bewahrt diese freilich nicht davor, entgegen ihrer Zurichtung verwendet zu werden. Bauteile werden mit Alltagsgegenständen identifiziert - ein Gebläse erinnert aufgrund eines angeschlossenen Schlauchs an einen „Staubsauger“; eine Glaskugel an „Weihnachtskugeln“. Geräte funktionieren nicht bzw. nicht wie gewünscht - entweder es muss nach Ersatz (z. B. 
Darstellung im Schulbuch) gesucht werden oder die Lehrer müssen erläutern, was sich eigentlich hätte zeigen sollen. Bisweilen konterkarieren die Schüler auch ganz bewusst die pädagogische „Zu-Mutung“ (Nohl 2011: 126), die ihnen in Gestalt der Dinge begegnet. Besonders auffällig ist dies im Zusammenhang mit Schülerexperimenten, denn gegen die ,Zumutungen' der Demonstrationsexperimente kann man sich allenfalls verbal (etwa durch ironische Zwischenrufe) wehren. Oft werden beispielsweise die Bestandteile von Experimentierkästen zweckentfremdet und beispielsweise dazu genutzt, Mitschüler zu ärgern. Dennoch erleichtert die didaktische Gestaltung der Lehrmittel ihren Einsatz als zu objektivierendem Unterrichtswerkzeug. Sie bieten der Objektivierung in ihrer Reduktion auf das Wesentliche nur geringen Widerstand und eignen sich deshalb für das hochgradig selektive disziplinäre Sehen.

Weder in der Lehrmittelindustrie noch in den Sammlungen dürfen die Dinge aber so zugerichtet werden, dass sie sich den Schülern vollständig und gänzlich eindeutig erschließen. Sie müssen als „epistemische Objekte" (Rheinberger 2006) hinreichend offen sein und Fragen aufwerfen. Anderenfalls finden die Versuche, die Dinge zu transformieren, keine Widerständigkeit, an der sie sich abarbeiten können. Eine disziplinäre Sicht ist notwendigerweise eine selektive Sicht, die etwas an den Dingen außer Acht lassen muss. Nur im Nicht-Identischen der unterschiedlichen Darstellungsformen kann sich an den Dingen auch etwas zeigen. Gerade deshalb kommt der praktischen Hervorbringung der Dinge als Objekte eine entscheidende Rolle in der schulischen Wissensvermittlung zu.

\section{Diskussion}

Die schulische Transformationsleistung erscheint zunächst als ein Abbild der Arbeit im naturwissenschaftlichen Labor. Zugleich zeigen sich aber deutliche Unterschiede. Anders als im Labor operiert der Schulunterricht nicht mit der Offenheit und Unsicherheit der Experimente. Kanonisiertes Wissen soll reproduziert werden. Dementsprechend abgesichert und eingeschränkt sind die Dinge in den schulischen Varianten der Naturwissenschaften. Auffällig ist im hier beschriebenen lehrerzentrierten Unterricht zunächst die asymmetrische Verteilung von Rede- und Manipulationsrechten in Bezug auf die Dinge. Die Lehrer sind diejenigen, die für die richtige Durchführung, Beobachtung und Versprachlichung der Experimente sorgen. Ihnen ge- bührt das alleinige Recht auf Manipulation ${ }^{3}$ und sie autorisieren sprachliche Benennung und schriftliche Mathematisierung. Die Schüler sind dagegen in auffälliger Weise auf distanzierte Sinne (Sehen, Hören) und beschränkte Teilnahme (Sprechen nach Aufforderung, Mit- bzw. Abschreiben) reduziert. Von der Vorbereitung der Dinge und Arrangements sind die Schüler gar gänzlich ausgeschlossen. All diese Maßnahmen sichern den fragilen Objektstatus der Dinge ab. Ein Einblick in die pannengefährdete Vorbereitung oder ein assoziatives Sprachspiel rund um das Experiment würden den linear sich zuspitzenden Ablauf der Objektivierung stören. Im ersten Fall ist der Status als didaktisches Werkzeug und der Inszenierungscharakter der Experimente zu offensichtlich - es darf sich nichts Überraschendes zeigen, sondern ausschließlich bereits Bekanntes. Im letzten Fall vervielfältigen sich die aufgerufenen Bedeutungen und Bezüge rund um das Ding. $\mathrm{Zu}-$ gleich können die Unterrichtsteilnehmer auf die curriculare Absicherung der sich in den Experimenten zeigenden Wissensbestände bauen. Wenn ein Experiment scheitert, dann gibt es alternative Vermittlungsformen: Unterrichtsgespräch, Schulbuch, Film, Simulation usw. Anders als in der Wissenschaft sind die Experimente auf maximale Deutlichkeit und Wahrnehmbarkeit ausgelegt. Während die Wissenschaftler im Labor tastend vorgehen und sich lange nicht sicher sind, ob sie etwas entdeckt haben, können die Schüler auf eine erprobte und verdichtete Wahrnehmbarkeit im Moment des Erkennens vertrauen. Die didaktische Zuspitzung naturwissenschaftlicher Experimente durch Lehrer und Lehrmittelindustrie garantiert ihnen dies.

Welche Schlüsse lässt die vorgestellte Forschung jenseits des konkreten Falls zu? Drei Bereiche sind hier betroffen:

(1) Mit dem mathematisch-naturwissenschaftlichen Schulunterricht ist eine gesellschaftliche Institution identifiziert, die als Schnittstelle zwischen Wissenschaft und Alltag fungiert. Naturwissenschaftliches Wissen findet über den Schulunterricht seinen Weg aus dem Labor in die Mitte der Gesellschaft. Der schulische Umgang mit diesem Wissen führt - vermittelt über die Dinge - zu einem verkürzten Modell wissenschaftlicher Erkenntnis. Durch die didaktische Reduktion und die sinnliche Distanzierung sind die Schüler in begrenzter Weise mit den Dingen konfrontiert. Der hier beschriebene Unter-

\footnotetext{
${ }^{3}$ Schülerexperimente sind hier ein Sonderfall. Allerdings ist dort der manipulative Umgang mit den Dingen stark reglementiert und durch das Design der Experimentierkästen begrenzt (siehe hierzu ausführlich Röhl 2013: 88-92).
} 
richt setzt auf eine "Zuschauertheorie des Erkennens“ (Dewey), bei der Gegenstände in „königlicher Abgeschiedenheit" vom erkennenden Geist erfasst werden (Balke 2008: 272). Die Schüler sind also nicht als wissenschaftliche Novizen vorgesehen, sondern als relativ passive Rezipienten eines durch Lehrmittel verkörperten, kanonisierten Wissens. Ferner wird hier eine Weltsicht eingeübt, die Subjekte und Objekte klar voneinander trennt und ihnen deutlich unterschiedliche Sphären zuweist: eine Sphäre der (sprachlichen) Bedeutung und des willentlichen Manipulierens und Beobachtens auf der einen und eine Sphäre des technischen Wirkens und der bloßen „Vorhandenheit“ (Heidegger 2006) auf der anderen Seite. Kultur- und wissenssoziologisch erweist sich der hier beschriebene lehrerzentrierte Schulunterricht dadurch als institutionalisierter Ort, an dem wir eine moderne Weltsicht einüben. Damit zeigt sich am Fall des Schulunterrichts, wo und wie naturwissenschaftliches Wissen jenseits des Labors tatsächlich wirksam sein kann und welche damit einhergehenden Annahmen für eine Soziologie alltäglichen Wissens relevant sein können.

(2) Im hier diskutierten lehrerzentrierten mathematisch-naturwissenschaftlichen Schulunterricht lernen die Schüler also nicht nur ein faktisches Wissen über ein Fach, sondern in zweierlei Hinsicht mehr: Zum einen lernen sie, eine spezifisch disziplinäre Transformation präsenter Dinge in die Form schriftlicher und graphischer Zeichen zu beherrschen; zum anderen werden sie in einer typisch „modernen“ Haltung zur Welt bestärkt. Damit weist die hier vorgestellte Studie auch den Weg zu einer Erforschung unterschiedlicher schulischer (Fach-)Kulturen und deren spezifische Transformationsleistung im Umgang mit Dingen. Wie gehen beispielsweise die Unterrichtsteilnehmer im Kunstunterricht mit Materialien und Werken um? Welche Haltung zu Dingen wird dadurch stark gemacht, dass man künstlerisch-schaffend mit Materialien umgeht? Welche Transformationen leistet man in offeneren, nicht-lehrerzentrierten Unterrichtsformaten? Damit verbunden ist auch jeweils die Frage nach den Spezifika des jeweiligen disziplinären Sehens und seiner Hervorbringung. Die Erweiterung der Unterrichtsforschung um die sozio-materielle Dimension ermöglicht damit einen weiteren $\mathrm{Zu}$ gang zum impliziten Wissen schulischen Unterrichtens. Ein Verdienst der qualitativen Unterrichtsforschung war es, die schulischen Wissensbestände jenseits des expliziten Fachwissens aufzuzeigen. Dies fand man vor allem in den impliziten Regeln der Interaktion. Nun kann man solche Wissens- bestände auch in den Dingen bzw. im Umgang mit ihnen aufsuchen. Auch hier gibt es einen „heimlichen Lehrplan“ (Zinnecker 1986), den es zu erforschen gilt. Was wir in der Schule lernen, lernen wir auch dadurch, dass wir mit und an Dingen etwas sehen.

(3) Darüber hinaus bietet der Aufsatz Anschlüsse für Diskussionen um die angemessene Berücksichtigung materieller Dinge in der Sozialtheorie. Es wurde deutlich, dass Dinge in der Praxis ganz unterschiedlich auftreten und relevant sein können. Insofern brauchen wir ein sozio-materielles Vokabular, das dieser Vielfalt gerecht wird. Nur während der Durchführung des Experiments kann man überhaupt von Handlungsträgerschaft - im kausalen Sinne (Rammert 2008: 356) - sprechen. Ansonsten bearbeiten Lehrer und Schüler sinnlich wahrnehmbare Eigenschaften der Dinge und nutzen diese als „kommunikative Ressource“ (Bergmann 1988). Einzelne gestaltete Elemente werden herausgegriffen und dienen als Bezugspunkte der Rezeption und des Gesprächs. Zentral ist im vorliegenden Fall also die sinnliche Gestalt(ung) der Dinge. Überlegungen aus der phänomenologischen Technikphilosophie (etwa Verbeek 2005) bieten hier vielversprechende Anschlussmöglichkeiten, da es nicht um ein ,hartes' kausales Wirken oder technische Zwänge, sondern um den wesentlich, weicheren' sinnlichen Aufforderungscharakter der Dinge geht. Damit stellt die hier vorgestellte sozio-materielle Perspektive auf den Schulunterricht auch einen Beitrag zu einer Soziologie der Dinge dar, die ein differenziertes Bild der Wirkmacht von Dingen zeichnen möchte.

\section{Schluss}

Dieser Aufsatz hat gezeigt, dass Dinge im lehrerzentrierten mathematisch-naturwissenschaftlichen Unterricht drei Phasen durchlaufen, die das Ergebnis praktischer Transformationen durch die Unterrichtsteilnehmer sind. Dinge sind zunächst Bestandteil eines vor allem nach curricularen Prinzipien geordneten schulischen Archivs. Sie müssen dort durch die Lehrer antizipierend in die situative Logik des Unterrichthaltens überführt werden. Im eigentlichen Unterricht treten die Dinge dann in Aktion. Die Unterrichtsteilnehmer arbeiten daran, dass die Dinge etwas fachlich Relevantes zeigen können und nehmen sich selbst dabei zurück. Schließlich werden die Dinge in die mediale Form von Sprache und Schrift überführt. Damit findet ein disziplinäres Sehen seinen Abschluss und die dargestellten Dinge 
erhalten den Status fachlicher Objekte. Dabei können die Unterrichtsteilnehmer auf die didaktische Gestaltung industriell gefertigter Lehrmittel zurückgreifen, die auf wesentliche Merkmale reduziert sind.

Darauf aufbauend konnte ferner dargelegt werden, dass erstens diese Form des Unterrichts auf die Einübung eines distanzierten (visuellen) Erkenntnismodells zielt, bei dem klar zwischen kulturellen Subjekten und materiellen Objekten unterschieden wird; dass es zweitens verschiedene Formen der disziplinären Wahrnehmung gibt, die praktisch hervorgebracht und angeleitet werden müssen; und dass man drittens zur Beschreibung solcher Transformationen auf ein differenziertes, auf den empirischen Fall abgestimmtes sozio-materielles Vokabular zurückgreifen muss.

\section{Literatur}

Balke, F., 2008: Was ist ein Ding? Zum Pragmatismus der neueren Wissenschaftsforschung. S. 269-283 in: A. Hetzel, J. Kertscher \& M. Rölli (Hrsg.), Pragmatismus. Philosophie der Zukunft. Weilerswist: Velbrück.

Balke, F., M. Muhle \& A. von Schöning (Hrsg.), 2012: Die Wiederkehr der Dinge. Berlin: Kadmos.

Bauersfeld, H., 1995: „Language Games“ in the Mathematics Classroom. Their Function and Their Effects. S. 271-292 in: P. Cobb \& H. Bauersfeld (Hrsg.), The Emergence of Mathematical Meaning. Interaction in Classroom Cultures. Hillsdale: Erlbaum.

Bergmann, J.R., 1988: Haustiere als kommunikative Ressourcen. S. 299-312 in: H.-G. Soeffner (Hrsg.), Kultur und Alltag (Soziale Welt Sonderband 6). Göttingen: Schwarz.

Bollig, S., H. Kelle \& R. Seehaus, 2012: (Erziehungs-)Objekte beim Kinderarzt. Zur Materialität von Erziehung in Kindervorsorgeuntersuchungen. S. 218-237 in: K. Priem, G.M. König \& R. Casale (Hrsg.), Die Materialität der Erziehung. Kulturelle und soziale Aspekte pädagogischer Objekte. Weinheim: Beltz.

Breidenstein, G., 2006: Teilnahme am Unterricht: Ethnographische Studien zum Schülerjob. Wiesbaden: VS.

Callon, M., 1986: Some Elements of a Sociology of Translation. Domestication of the Scallops and the Fishermen of St Brieuc Bay. S. 196-223 in: J. Law (Hrsg.), Power, Action and Belief. A New Sociology of Knowledge? London: Routledge.

Coole, D. \& S. Frost, 2010: New Materialisms. Ontology, Agency, and Politics. Durham: Duke University Press.

Dant, T., 2004: The Driver-Car. Theory, Culture \& Society $21: 61-79$.

Doering, H. \& S. Hirschauer, 1997: Die Biographie der Dinge. Eine Ethnographie musealer Repräsentationen. S. 267-297 in: S. Hirschauer \& K. Amann (Hrsg.), Die Befremdung der eigenen Kultur. Frankfurt a.M.: Suhrkamp.
Dorr-Bremme, D.W., 1990: Contextualization Cues in the Classroom. Discourse Regulation and Social Control Functions. Language in Society 19: 379-402.

Eberbach, C. \& K. Crowley, 2009: From Everyday to Scientific Observation. How Children Learn to Observe the Biologist's World. Review of Educational Research 79: 39-68.

Erlemann, M., 2004: Inszenierte Erkenntnis. Zur Wissenschaftskultur der Physik im universitären Lehrkontext. S. 53-90 in: M. Arnold \& R. Fischer (Hrsg.), Disziplinierungen. Kulturen der Wissenschaften im Vergleich. Wien: Turia + Kant.

Fenwick, T. \& R. Edwards, 2010: Actor-Network Theory in Education. London: Routledge.

Fodor, J., 1984: Observation Reconsidered. Philosophy of Science 51: 23-43.

Frers, L., 2007: Einhüllende Materialitäten. Eine Phänomenologie des Wahrnehmens und Handelns an Bahnhöfen und Fährterminals. Bielefeld: transcript.

Garfinkel, H., M. Lynch \& E. Livingston, 1981: The Work of a Discovering Science Construed with Materials from the Optically Discovered Pulsar. Philosophy of the Social Sciences 11: 131-158.

Goffman, E., 1980: Rahmen-Analyse. Ein Versuch über die Organisation von Alltagserfahrungen. Frankfurt a.M.: Suhrkamp.

Goodwin, C., 1994: Professional Vision. American Anthropologist 96: 606-633.

Greiffenhagen, C., 2014: The Materiality of Mathematics. Presenting Mathematics at the Blackboard. British Journal of Sociology 65: 502-528.

Gugutzer, R., 2012: Verkörperungen des Sozialen. Neophänomenologische Grundlagen und soziologische Analysen. Bielefeld: transcript.

Gurwitsch, A., 1974: Phenomenology and the Theory of Science. Evanston: Northwestern University Press.

Heidegger, M., 1959: Das Ding. S. 163-181 in: Vorträge und Aufsätze. Pfullingen: Neske.

Heidegger, M., 2006: Sein und Zeit. Tübingen: Niemeyer.

Hindmarsh, J. \& C. Heath, 2000: Sharing the Tools of the Trade. The Interactional Constitution of Workplace Objects. Journal of Contemporary Ethnography 29: 523-562.

Hnilica, S., 2010: Schulbank und Klassenzimmer. Disziplinierung durch Architektur. S. 141-162 in: R. Egger \& B. Hackl (Hrsg.), Sinnliche Bildung? Pädagogische Prozesse zwischen vorprädikativer Situierung und reflexivem Anspruch. Wiesbaden: VS.

Hörning, K.H., 2001: Experten des Alltags. Die Wiederentdeckung des praktischen Wissens. Weilerswist: Velbrück.

Hörning, K.H., 2005: Lob der Praxis. Praktisches Wissen im Spannungsfeld zwischen technischen und sozialen Uneindeutigkeiten. S. 297-310 in: G. Gramm \& A. Hetzel (Hrsg.), Unbestimmtheitssignaturen der Technik. Eine neue Deutung der technisierten Welt. Bielefeld: transcript.

Ihde, D., 1990: Technology and the Lifeworld. From Garden to Earth. Bloomington: Indiana University Press.

Ingold, T., 2007: Materials Against Materiality. Archaeological Dialogues 14: 1-16. 
Introna, L., F. Ilharco \& E. Fay (Hrsg.), 2008: Phenomenology, Organisation and Technology. Lissabon: Universidade catolica editora.

Kalthoff, H., 1997: Wohlerzogenheit. Eine Ethnographie deutscher Internatsschulen. Frankfurt a.M.: Campus.

Kalthoff, H., 2003: Die beobachtende Differenz. Instrumente der ethnografisch-soziologischen Forschung. Zeitschrift für Soziologie 32: 70-90.

Kalthoff, H., T. Cress \& T. Röhl (Hrsg.), 2015: Materialität. Herausforderungen für die Sozial- und Kulturwissenschaften. Paderborn: Fink.

Knoblauch, H. \& C. Heath, 2006: Die Workplace Studies. S. 141-161 in: W. Rammert \& C. Schubert (Hrsg.), Technografie. Zur Mikrosoziologie der Technik. Frankfurt a.M.: Campus.

Knorr-Cetina, K., 1984: Die Fabrikation von Erkenntnis. Zur Anthropologie der Naturwissenschaft. Frankfurt a.M.: Suhrkamp.

Knorr-Cetina, K., 1989: Spielarten des Konstruktivismus. Einige Notizen und Anmerkungen. Soziale Welt 40: 86-96.

Knorr-Cetina, K., 2007: Umrisse einer Soziologie des Postsozialen. S. 25-39 in: H. Pahl \& L. Meyer (Hrsg.), Kognitiver Kapitalismus. Soziologische Beiträge zur Theorie der Wissensökonomie. München: Metropolis.

Kopytoff, I., 1986: The Cultural Biography of Things: Commoditization as Process. S. 64-94 in: A. Appadurai (Hrsg.), The Social Life of Things. Commodities in Cultural Perspective. Cambridge: Cambridge University Press.

Krummheuer, G., 2002: Der mathematische Anfangsunterricht. Weinheim: Beltz.

Langeveld, M.J., 1955: Das Ding in der Welt des Kindes. Zeitschrift für Pädagogik 1: 69-83.

Latour, B., 2001: Eine Soziologie ohne Objekt? Anmerkungen zur Interobjektivität. Berliner Journal für Soziologie 11: 237-252.

Latour, B., 2007: Eine neue Soziologie für eine neue Gesellschaft. Frankfurt a.M.: Suhrkamp.

Latour, B., 2008: Wir sind nie modern gewesen. Versuch einer symmetrischen Anthropologie. Frankfurt a.M.: Suhrkamp.

Latour, B. \& S. Woolgar, 1979: Laboratory Life. Princeton: Princeton University Press.

Lindemann, G., 2009: Das Soziale von seinen Grenzen her denken. Weilerswist: Velbrück.

Lüders, J. (Hrsg.), 2007: Fachkulturforschung in der Schule. Opladen: Barbara Budrich.

Lynch, M., E. Livingston \& H. Garfinkel, 1985: Zeitliche Ordnung in der Arbeit des Labors. S. 179-206 in: W. Bonß \& H. Hartmann (Hrsg.), Entzauberte Wissenschaft (Soziale Welt Sonderband 3). Göttingen: Schwarz.

Lynch, M. \& D.H. Macbeth, 1998: Demonstrating Physics Lessons. S. 269-297 in: J.G. Greeno \& S.V. Goldman (Hrsg.), Thinking Practices in Mathematics and Science Learning. Mahwah: Erlbaum.

Mehan, H., 1979: Learning Lessons. Social Organization in the Classroom. Cambridge, Mass.: Harvard University Press.
Merleau-Ponty, M., 1976: Phänomenologie der Wahrnehmung. Berlin: de Gruyter.

Meyer-Drawe, K., 1999: Herausforderung durch die Dinge. Das Andere im Bildungsprozeß. Zeitschrift für Pädagogik 45: 329-342.

Mollenhauer, K., 1991: Vergessene Zusammenhänge. Über Kultur und Erziehung. Weinheim: Juventa.

Nespor, J., 1994: Knowledge in Motion. Space, Time and Curriculum in Undergraduate Physics and Management. London: Routledge.

Neumann, S., 2012: Pädagogisierung und Verdinglichung. Beobachtungen zur Materialität der Frühpädagogik. S. 168-184 in: K. Priem, G.M. König \& R. Casale (Hrsg.), Die Materialität der Erziehung. Kulturelle und soziale Aspekte pädagogischer Objekte. Weinheim: Beltz.

Nohl, A.-M., 2011: Pädagogik der Dinge. Bad Heilbrunn: Klinkhardt.

Nohl, A.-M. \& C. Wulf (Hrsg.), 2013: Mensch und Ding Die Materialität pädagogischer Prozesse. Wiesbaden: VS.

Oelkers, J., 2010: Lehrmittel. Rückgrat des Unterrichts. Folio 135: 18-21.

Peirce, C.S., 1983: Phänomen und Logik der Zeichen. Frankfurt a.M.: Suhrkamp.

Piaget, J., 1992: Das Weltbild des Kindes. München: DTV.

Priem, K., G.M. König \& R. Casale (Hrsg.), 2012: Die Materialität der Erziehung. Kulturelle und soziale Aspekte pädagogischer Objekte. Weinheim: Beltz.

Rammert, W., 2008: Technographie trifft Theorie. Forschungsperspektive einer Soziologie der Technik. S. 341-367 in: H. Kalthoff, S. Hirschauer \& G. Lindemann (Hrsg.), Theoretische Empirie. Zur Relevanz qualitativer Forschung. Frankfurt a.M.: Suhrkamp.

Rammert, W. \& I. Schulz-Schaeffer, 2002: Technik und Handeln. Wenn soziales Handeln sich auf menschliches Verhalten und technische Artefakte verteilt. S. 11-64 in: W. Rammert \& I. Schulz-Schaeffer (Hrsg.), Können Maschinen handeln? Frankfurt a.M.: Campus.

Rheinberger, H.-J., 2006: Experimentalsysteme und epistemische Dinge. Eine Geschichte der Proteinsynthese im Reagenzglas. Frankfurt a.M.: Suhrkamp.

Röhl, T., 2013: Dinge des Wissens. Schulunterricht als sozio-materielle Praxis. Stuttgart: Lucius \& Lucius.

Schindler, L., 2009: The Production of „Vis-Ability“. An Ethnographic Video Analysis of a Martial Arts Class. S. 135-153 in: U.T. Kissmann (Hrsg.), Video Interaction Analysis. Methods and Methodology. Frankfurt a.M.: Lang.

Schubert, C., 2011: Die Technik operiert mit. Zur Mikroanalyse medizinischer Arbeit. Zeitschrift für Soziologie 40: 174-190.

Schulz, M., 2013: Frühpädagogische Konstituierung von kindlichen Bildungs- und Lernprozessen. Zeitschrift für Soziologie der Erziehung und Sozialisation 33: 26-41.

Sørensen, E., 2009: The Materiality of Learning. Technology and Knowledge in Educational Practice. Cambridge: Cambridge University Press.

Stieve, C., 2008: Von den Dingen lernen. Die Gegenstände unserer Kindheit. München: Fink. 
Ten Have, P., 2004: Understanding Qualitative Research and Ethnomethodology. London: Sage.

Verbeek, P.-P., 2005: What Things Do. Philosophical Reflections on Technology, Agency, and Design. University Park: Penn State Press.

Waldenfels, B., 2006: Phänomenologie und Phänomenotechnik. S. 367-380 in: J. Jonas \& K.-H. Lembeck (Hrsg.), Mensch - Leben - Technik. Aktuelle Beiträge zur phänomenologischen Anthropologie. Würzburg: Königshausen \& Neumann.
Wiesemann, J. \& J. Lange, 2014: Wissen schaffen durch die Dinge? Ergebnisse aus einer ethnographischen Studie zur Materialität im Sachunterricht. Zeitschrift für Grundschulforschung 7: 46-59.

Willems, K., 2007: Schulische Fachkulturen und Geschlecht. Physik und Deutsch - natürliche Gegenpole? Bielefeld: transcript.

Zinnecker, J. (Hrsg.), 1986: Der heimliche Lehrplan. Untersuchungen zum Schulunterricht. Weinheim: Beltz.

\section{Autorenvorstellung}

Tobias Röhl, geb. 1978 in Husum. Studium der Soziologie, Kunst- und Medienwissenschaft sowie Sprachwissenschaft mit anglistischem Schwerpunkt in Konstanz und Dublin. Promotion 2012 in Mainz. Von 2006-2008 wissenschaftlicher Mitarbeiter an der Universität Konstanz; seit 2008 wissenschaftlicher Mitarbeiter an der Johannes Gutenberg-Universität Mainz.

Forschungsschwerpunkte: Wissens- und Bildungssoziologie, materielle Kultur, qualitative Methoden der Sozialforschung.

Wichtigste Publikationen: Dinge des Wissens. Schulunterricht als sozio-materielle Praxis, Stuttgart 2013; Interobjectivity and Interactivity. Material Objects and Discourse in Class (mit Herbert Kalthoff), in: Human Studies 34, 2011. 